\title{
Médiévales
}

Langues, Textes, Histoire

47 | automne 2004

Îles du Moyen Âge

\section{La voie des îles}

The Pathway of Islands

\section{Frank Lestringant}

\section{(2) OpenEdition}

Journals

Édition électronique

URL : https://journals.openedition.org/medievales/506

DOI : 10.4000/medievales.506

ISSN : $1777-5892$

\section{Éditeur}

Presses universitaires de Vincennes

\section{Édition imprimée}

Date de publication : 1 décembre 2004

Pagination : 113-122

ISBN : 2-84292-157-7

ISSN : 0751-2708

\section{Référence électronique}

Frank Lestringant, "La voie des îles », Médiévales [En ligne], 47 | automne 2004, mis en ligne le 02 septembre 2006, consulté le 24 avril 2022. URL : http://journals.openedition.org/medievales/506 ; DOI : https://doi.org/10.4000/medievales.506

Ce document a été généré automatiquement le 24 avril 2022

Tous droits réservés 


\title{
La voie des îles
}

\author{
The Pathway of Islands
}

\section{Frank Lestringant}

1 Cet épilogue sera comme un îlot ultime raccroché à l'archipel des îles médiévales. Ou comme un pont d'îles jeté entre le Moyen Âge et la Renaissance. - Je pense à la chaussée d'Adam, reliant le pays tamoul à Ceylan, et à cet incroyable chemin de fer posé sur l'eau sur des dizaines de milles, impraticable à la moindre tempête.

2 Que conclure d'un si riche ensemble de contributions? D'abord, peut-être, qu'il faut distinguer entre le mot et la chose, l'objet géographique et le concept. Une île, en soi, n'a rien de bien extraordinaire. Elle ne se distingue de toute autre terre que par sa séparation. Dans le cours de l'histoire, l'île connaît les mêmes vicissitudes que le continent qu'elle avoisine. À preuve les îles de la mer Égée au cours d'une longue fin de Moyen Âge sanglante et mouvementée, théâtre de l'affrontement séculaire entre les deux chrétientés, puis entre chrétiens et musulmans. Dans le Péloponnèse, en Dalmatie ou en Afrique du Nord, des enclaves de terre ferme se comportent comme des îles. Henri Bresc parle à ce propos d'un "archipel de niches ", des poches de contrôle fortifiées et ravitaillées par la mer s'établissant sur les points remarquables de la côte ${ }^{1}$. Du point de vue économique non plus, rien ne distingue les enclaves continentales de l'archipel des spécialisations insulaires : ici la vigne et là l'olivier, le blé de Sicile et la canne à sucre de Valence. Réciproquement, la mer ne constitue pas une entité spécifique. En témoigne là encore la mer Égée. Située pendant des siècles à la jonction de deux mondes antagonistes, ou plutôt au point de rencontre et de chevauchement de deux "plaques" tout à la fois architectoniques et humaines, la chaîne des îles de l'Archipel se comporte comme n'importe quel front, à peine plus mobile ou plus perméable qu'une frontière terrestre ${ }^{2}$.

Mondes miniatures

3 Tout au plus, l'île offre à l'historien un modèle réduit où les flux se déroulent en accéléré : plus vite razziée et dévastée, plus vite vidée de ses habitants massacrés ou déportés, et plus vite reconstruite et repeuplée. Ainsi des Sporades et des Cyclades durant l'affrontement de plusieurs siècles entre la chrétienté et l'islam; ainsi des Grandes Antilles touchées les premières par le choc démographique de la Conquista, 
réduites en quinze ans à peine à une table rase, sur laquelle s'édifie peu après la nouvelle société coloniale. Pour l'anthropologue ou l'historien, l'île est un laboratoire où se vérifient à l'œil nu, dans un temps et un espace restreints, des phénomènes ailleurs plus diffus, plus complexes et plus lents. Tel est le mérite des îles et aussi leur limite. Elles éclairent et elles déçoivent. On constate l'extrême volatilité des économies insulaires ${ }^{3}$. On goûte le spectacle bigarré et changeant des îles. On admire leur floraison, mais l'on s'étonne ensuite de leur effacement ou de leur désertification, après des décennies de prospérité. Feu de paille, feu d'îles. Au temps de Jules Verne encore, l'Archipel est en feu, tardant à renaître de ses cendres à l'issue de la guerre d'indépendance grecque ${ }^{4}$.

Mondes à part

4 Mais le plan du réel n'est pas le seul qu'il faille prendre en considération. Les métamorphoses de l'île ne s'arrêtent pas à la surface du globe ni à la succession objective des événements. Il faut aussi considérer les plans symbolique et imaginaire.

5 Le plan symbolique tout d'abord. Depuis Homère et Hésiode, les îles reçoivent un traitement à part dans le savoir géographique, et pas seulement parce qu'elles sont des réservoirs de mythes. C'est un usage ancien que de les regrouper, et de les débiter par catalogue, à la manière des vaisseaux des Grecs au chant II de l'Iliade. Semblables dénombrements se rencontrent chez Strabon et Ptolémée ${ }^{5}$. Diodore de Sicile consacre aux îles un livre particulier de sa Bibliothèque historique, le livre $\mathrm{V}$ où il passe en revue les îles les plus importantes de la Méditerranée et de l'Océan, en commençant par la Sicile. Ce livre d'îles (nesiotiké) peut être considéré comme le premier Isolario à proprement parler de la littérature classique ${ }^{6}$. Le point de vue est à dominante mythographique, les îles étant pour Diodore les lieux privilégiés où s'est opérée la rencontre extraordinaire entre les dieux et les hommes, à preuve Chypre où Aphrodite est née de l'écume de la mer, Délos ou Naxos, théâtre des amours d'Ariane et de Dionysos. C'est dans les îles qu'ont été recueillis les premiers bienfaits de la civilisation ${ }^{7}$. À l'exemple de Diodore, Pline l'Ancien consacre une section particulière de son Histoire naturelle aux îles, à leur naissance, à leur disparition et à leurs incessantes métamorphoses ${ }^{8}$. Dans la Chorographie de Pomponius Mela et dans la Périégèse en vers de Denys d'Alexandrie, toutes les îles - respectivement de la Méditerranée et de l'oekoumène - sont décrites dans un chapitre particulier. Des catalogues des îles les plus grandes, limitées à la Méditerranée et arrêtées au chiffre canonique de sept, se retrouvent dans la tradition historiographique (Timée, Appien) et dans la comédie moyenne`. À l'époque hellénistique, la distinction îles-continent répond à des fins didactiques et scolaires.

6 Le premier des modernes à concevoir le programme d'une description de l'univers insulaire est Domenico Silvestri, un notaire florentin ami de Coluccio Salutati. Son De Insulis et earum proprietatibus, composé à la fin du XIVe siècle, se propose de combler une lacune du De montibus de Boccace, lequel traitait des montagnes, des lacs et des fleuves, mais oubliait les îles ${ }^{10}$. Et pourtant ces dernières, explique Silvestri, sont d'un grand intérêt, en raison de leurs qualités singulières: elles naissent et disparaissent, se déplacent et changent de nom avec une déconcertante facilité. Au début du XV siècle enfin est composé le fameux Liber insularum Archipelagi du prêtre florentin Cristoforo Buondelmonti, modèle du "livre des îles " que va constituer, pendant près de trois siècles, le genre de l'Isolario ou atlas insulaire. La forme canonique en est donnée par 
Benedetto Bordone, auteur en 1528 du Libro de tutte l'Isole del Mondo, réédité en 1534 sous le titre définitif d'Isolario ${ }^{11}$.

7 Leur différence de situation, de dessin et de nature (constituées de terre, elles se définissent par l'eau qui les entoure) explique que les îles doivent être traitées à part des continents. De plus, les îles entraînent une disposition particulière de l'exposé. Décrire les îles du monde, ou, mieux, décrire le monde par îles implique un émiettement de la matière géographique et aussi une plus grande liberté dans sa mise en ordre. L'archipel peut être pris à partir de n'importe quel bout et dévidé à volonté, selon les parcours les plus variés.

Du monde clos au monde ouvert

Comme Domenico Silvestri s'en émerveillait et s'en désolait tout à la fois, il est impossible de dénombrer les îles. L'inachèvement de l'archipel constitue en vérité une formidable richesse. C'est par les îles, élément mouvant et malléable, que le monde clos du Moyen Âge s'est ouvert et renouveléc ${ }^{12}$. Dans une imago mundi figée et fixée depuis des siècles, les îles du pourtour océanique contenaient la possibilité d'une expansion; elles recelaient un dynamisme latent. Si le monde de la Renaissance a pu faire craquer ses coutures, c'est parce qu'à la périphérie des trois continents connus il tendait déjà à s'émietter. L'archipel circulaire aménageait aux limites de l'oekoumène un espace incertain et mouvant où le rêve était tout près de se concrétiser. Il n'y fallait que de l'audace et des moyens techniques d'abord insoupçonnés.

9 Le dynamisme des îles est inhérent à leur instabilité et à leur impossible dénombrement. L'île est mobile comme le navire ou la bouée dérivante, comme l'esturgeon ou le poisson Jasconius, sur le dos duquel chaque dimanche de Pâques pendant sept ans le bon abbé saint Brandan célèbre la messe. Amas d'îles au pourtour de la mappemonde, l'archipel océanique est aussi meuble que le sable du littoral ou la poussière d'étoiles du firmament. Disséminées, éparpillées, ces réserves d'imaginaire échappaient à la prise, fuyaient entre les doigts. Pourtant leur capture et leur réunion en un agglomérat solide n'étaient qu'une affaire de temps.

10 Le dynamisme de l'expansion latérale de l'Insulaire, au départ, est lent. De la timidité des premiers tâtonnements témoigne le De Canaria de Boccace. Les découvreurs se tiennent sur la réserve. L'archipel des Canaries abonde en merveilles, mais déjà il déçoit, comme le premier aperçu des Antilles va décevoir Colomb, lors du voyage inaugural de 1492-1493. Peu de ressources, des peuples pauvres et démunis des arts de la civilisation, ignorant de surcroît les métaux précieux qu'on leur montre. La curiosité des visiteurs, faute de mieux, se concentre pour finir sur les hommes. L'île, nous dira Leandro Alberti dans sa Descrittione di tutta Italia, est le lieu idéal de la description anthropologique, parce que circonscrit et préhensible, ramenant le regard vers l'humain et le singulier, d'un côté les humbles travaux des saisons et des jours, de l'autre les célébrités locales, les hommes illustres natifs de l'endroit ${ }^{13}$. L'éloge de l'île se rapproche de l'éloge de ville, comme le veut la paronomase. îles et villes se confondent du reste souvent dans les périples méditerranéens. Nombre d'îles grecques se réduisent à leur ville principale, à leur port, à l'escale. Cette confusion est renforcée «par la toponymie qui donne fréquemment à la ville principale le nom de l'île (Corfou, Rhodes, Chios, Candie...) et par la perception maritime qui rend parfois difficile la distinction entre terre insulaire et terre continentale $»^{14}$.

11 Rien de tel dans l'archipel atlantique. Ces îles nouvelles sont sans villes et sans monuments, sans écriture et sans mémoire. De surcroît elles sont pauvres. Les quelques 
merveilles qu'on trouve aux Canaries compensent mal la déconvenue initiale. Était-ce donc cela, les îles Fortunées chantées par les Anciens ? Force est de se rabattre sur ce qu'on trouve : une idole de pierre représentant un homme nu tenant un globe dans sa main, des peaux de boucs et de chèvres, du suif, de l'huile de poisson, des peaux de phoques et du bois rouge à teindre les étoffes, qui n'est même pas l'authentique bois brésil. Pour compléter ce misérable butin, on se saisit de quelques indigènes que l'on ramène de force en Europe, à titre de curiosités exotiques. Tout l'avenir de la conquête du Nouveau Monde est écrit là par avance, pour ainsi dire, entre émerveillement et déconvenue, accaparement avide et incompréhension. Mais il y aura en prime, pour Cortés ou Pizarre, les montagnes et les mines, l'or du Mexique et l'argent du Potosi.

Le temps des îles, qui s'étire tout au long du $\mathrm{XV}^{\mathrm{e}}$ siècle, sera suivi d'un temps des continents. Les îles à la Renaissance vont progressivement perdre de leur prestige, dès lors qu'elles apparaissent comme le simple prélude à de plus amples découvertes. Dans l'éloge de la navigation qu'il place en tête de son livre des Singularitez de la France Antarctique, en 1557, le cosmographe André Thevet rappelle qu'il ne faut pas s'arrêter à la surface de la terre «triste, et mélancolique, couverte le plus souvent de pierres, épines et chardons ", mais l'ouvrir et la sonder, comme fait le laboureur, lequel, « avec soc et charrue ", en tire "grand émolument $»^{15}$. De même, les navigateurs modernes ont su ne pas s'arrêter perpétuellement aux îles « inconstantes, et mal assurées », pour atteindre finalement « la terre ferme, bonne, et fertile».

L'opposition est géographique, mais aussi morale : contrairement aux îles inconstantes, la terre ferme tient ses promesses. D'où la supériorité des Modernes sur les Anciens. Ces derniers ont certes labouré la mer, mais ils n'ont guère dépassé le mur évanescent des îles, comme le montre l'exemple d'Ulysse. Seuls les Modernes, laissant loin derrière eux toute balise flottante, ont eu accès à la terre ferme située au-delà de la ceinture océane et ont recueilli alors des profits à la mesure de leurs espoirs et de leurs peines. Car le marin, s'avançant plus avant que le laboureur, pénètre tour à tour deux sols ou deux écorces: d'une part l'étendue liquide que fend l'étrave du navire, de l'autre le voile flottant à l'horizon des îles-fantômes qui dérobent à la vue la réalité des mondes nouveaux ${ }^{16}$.

Îles verticales

Il ne faut pourtant pas désespérer des îles. Ni surtout en médire. Car elles n'ouvrent pas seulement sur des ailleurs à fleur de terre, une transcendance horizontale en quelque sorte, ramifiée selon les vingt-quatre aires de vent de la rose. Le dynamisme des îles est aussi un dynamisme vertical. Sas entre les étages de la Création, l'île ouvre vers les gouffres et simultanément vers les étoiles. Tantôt c'est la bouche de l'Enfer ou son antichambre, les Limbes remplis d'enfants vagissants et de marins engloutis sans sépulture : on connait le Purgatoire de saint Patrick situé dans une île du Lough Derg ou lac Rouge en Irlande, lieu de pénitence et objet de pèlerinage depuis le $\mathrm{XII}^{\mathrm{e}}$ siècle $^{17}$; l'Irlande a pour sœur l'Islande avec ses monts fumants et vomissants, autre porte des royaumes infernaux, comme la peindra Olaus Magnus, archevêque d'Uppsal, dans sa Carta marina de $1539^{18}$. Tantôt c'est une montagne inaccessible qui porte tout au haut de ses pentes abruptes, segmentées par d'étroites terrasses, le paradis terrestre où règne un éternel été : tel est dans son élan vertigineux le Purgatoire de Dante, dressé au milieu de l'océan austral, pic des antipodes dont l'aiguille parcourt de son ombre l'hémisphère inverse du nôtre. 
15 Le pic du Purgatoire - mais la même chose pourrait se dire d'un cône volcanique accomplit simultanément la remontée vers l'origine et l'ascension vers le terme, un parcours en amont vers les racines de la terre et de l'histoire, et en aval, vers les fins dernières et la vie céleste.

On le voit encore dans les Isolari de la Renaissance, de Benedetto Bordone à André Thevet. Dans ces atlas d'îles, la représentation figurée renonce aux conventions cartographiques habituelles. À la vue d'en haut est préférée la vue cavalière, ou plutôt la vision depuis la hune du vaisseau, qui creuse les reliefs, dégage des aspérités et amplifie les profondeurs : les îles de la mer Égée se fendent et s'entrouvrent jusqu'à leur socle, éclatent en grottes et en gouffres. La surface étale des choses disposées selon méridiens et parallèles est alors sacrifiée aux élans verticaux d'une topographie qui n'exagère le relief d'infimes îlots que pour faire entrer en carte les réserves d'imaginaire contenues dans leur sous-sol. Par ces cheminées actives qui trouent la Méditerranée, un paysage archaïque remonte au jour, anticipant à plusieurs siècles d'intervalle la géographie « extraordinaire » d'un Jules Verne.

On ne s'étonnera donc pas si plus tard, face au Labrador, surgit une île des Démons survolée par des diables griffus aux jambes de bouc, comme les représentera Giacomo Gastaldi en 1548, dans une carte que l'érudit Giovanni Battista Ramusio insèrera quelques années après dans son recueil des Navigationi \& viaggi.

Les trous du récit

18 L'île a enfin une fonction narrative. Dans le récit écrit ou oral, le motif de l'île introduit mobilité et fragmentation. Il aménage des pauses et des haltes dans une narration qui pourrait à la longue lasser le lecteur ou l'auditeur. Il favorise les techniques de l'incrustation et de l'inclusion. Au chant XVIII de l'Iliade, le bouclier d'Achille forgé par Héphaïstos est rond comme le monde et rond comme une île. L'Océan entoure le bouclier comme il entoure la terre : "Héphaïstos mit encore Sa Grande Force le fleuve Océan sur le bord extrême du bouclier solidement fait ». C'est l'ornement de la dernière bande, la clôture qui renferme le tout ${ }^{19}$. Depuis Homère, l'île est le lieu idéal de l'ekphrasis: le bouclier d'Achille dans l'Iliade, les récits d'Ulysse chez Alkinoos dans l'Odyssée. C'est un trou - ou au contraire un relief - dans le texte. S'y loge sans difficulté un récit secondaire, plus ou moins bien raccordé au récit principal, comme le montre encore, au XIII ${ }^{\mathrm{e}}$ siècle, l'Estoire del saint $\mathrm{Graal}^{20}$. Dans l'écoulement narratif du roman ou de la geste héroïque, l'île représente une pause après les combats et les tempêtes, l'étape où l'aède reprend souffle et force pour chanter d'autres histoires, aborder à d'autres rivage, franchir le seuil d'autres époques, ou parler tout simplement de son art et résumer l'œuvre en cours.

19 Là encore l'île est un sas. Un sas ouvrant sur les univers parallèles du mythe ou de la légende. Thomas More, à l'aube du XVI ${ }^{\mathrm{e}}$ siècle, l'a très bien compris, qui situe l'Utopie dans une île, sur la toile de fond des découvertes espagnoles et portugaises. Érasme fera naître dame Folie, dont il écrit l'Éloge, dans les îles Fortunées, celles de la mythologie plutôt que celles des modernes navigateurs et géographes, de Béthencourt à Pétrarque. Nulle difficulté pour loger dans l'île la république idéale, ou pour y peindre l'humanité restée à l'état de nature. Comme le montre Antoine Franzini, il n'a pas fallu pour cela attendre les grandes navigations et la découverte des Moluques et des Antilles. Pour Antonio Ivani ou Pietro Cirneo, la Corse fait très bien l'affaire. Le laboratoire insulaire était à portée de main, et les humanistes du Quattrocento le découvrent avec émerveillement. S'y dessine d'emblée l'alternative entre la colonisation douce, dans le 
respect des coutumes locales et de la liberté native des insulaires, et la soumission par la contrainte d'un peuple sauvage qu'il faut au préalable domestiquer pour mieux l'éduquer. On sait que ce choix commandera le destin du Nouveau Monde et de son exploitation, et que le pire prévaudra.

Le sas de l'île ouvre pour finir sur la réflexivité et sur l'ironie. Thomas More le prouve encore, avec sa critique détournée de l'Angleterre et des sociétés réelles par Raphaël Hythloday, le "conteur de sornettes", le voyageur au long cours revenu d'Utopie, c'est-à-dire de « nulle part ». Vis-à-vis du continent des certitudes, stable et arrêté, l'île fuyante, l'île improbable représente la part du jeu et du défi intellectuel, le lieu mobile à partir duquel remettre en cause les tutelles écrasantes, les idées reçues, les dogmes. C'est pourquoi, tout comme le Moyen Âge, tout comme la Renaissance, notre temps de globalisation a besoin des îles.

\section{NOTES}

1. Voir ici même H. Bresc, « Îles et tissu connectif de la Méditerranée médiévale ».

2. Voir ici même les communications de G. Saint-Guillain et de P. F. Simbula.

3. H. Bresc, art. cit.

4. J. Verne, L'Archipel en feu, Paris, 1884.

5. Voir J. M. Montesdeoca Medina, «Del Enciclopedismo grecolatino a los Islarios humanistas. Breve historia de un genero ", Revista de Filologia, 19, enero 2001, p. 229-253.

6. Voir F. Prontera, « Géographie et mythes dans 1'“isolario" des Grecs ", in M. Pelletier éd., Géographie du Monde au Moyen Âge et à la Renaissance, Paris, 1989, p. 169-179. Cf.

M. Donattini, Spazio e modernità. Libri, carte, isolari nell'età delle scoperte, Bologne, 2000, p. 171.

7.F. PRONTERA, art. cit., p. 179.

8.Pline l'Ancien, Histoire naturelle, trad. par Antoine Du Pinet sous le titre de L'Histoire du Monde de C. Pline Second, Lyon, 1562 et 1581, livre II, chap. LXXXVI : « Des nouvelles Isles qui surgissent en la mer »; LXXXVII : « Des Isles advenues par adventure »; LXXXIX : «Des Isles reunies à la terre ferme »; XC : « Des terres peries par eau »; XCI : « Des terres qui se sont englouties, et abysmées elles mesmes »; XCII : « Des villes englouties et abysmées par la mer »; XCV : « Des Isles qui ne sont fermes, ains flottent sur l'eau ». 9. F. PRONTERA, art. cit., p. 173.

10. Voir sur ce point M. Donattini, Spazio e modernità, op. cit., p. 172.

11. B. Bordone, Isolario di Benedetto Bordone Nel qual si ragiona di tutte l'Isole del mondo, con li lor nomi antichi \& moderni, historie, favole, \& modi del loro vivere, \& in qual parte del mare stanno, \& in qual parallelo \& clima giaciono. Con la gionta del Monte del Oro novamente ritrovato. Con il Breve del Papa, Venise, 1534 ; édition en fac-similé, préface d'Umberto Eco, Paris-Turin, 2000.

12. Comme le rappelle ici Nathalie Bouloux.

13. L. Alberti, Isole appartenenti alla Italia, di F. Leandro Alberti Bolognese, Venise, 1567, $\mathrm{f}^{\circ} 4 \mathrm{r}^{\circ}$. 
14. M.-H. Prat et P. Servet, « La description des îles du Levant dans la littérature géographique de la Renaissance ", dans L. Zilli éd., L'Europa e il Levante nel Cinquecento. II. Le Isole del Mediterraneo orientale nella letteratura di viaggio, Padoue, 2004, p. 33-88 ; cit., p. 51.

15. A. Thevet, Les Singularitez de la France Antarctique, autrement nommée Amerique, Paris, 1557 et 1558 , chap. $1^{\text {er }}$ : « L'embarquement de l'auteur », fo $1 v^{0}$; éd. F. Lestringant, Paris, 1997, p. 43-44.

16. J'ai développé ce point dans Le Livre des îles. Atlas et récits insulaires de la Genèse à Jules Verne, Genève, 2002, p. 145-146.

17. J. Le Goff, La naissance du Purgatoire, Paris, 1981, p. 268.

18. O. Magnus, Carta Marina et descriptio septemtrionalium terrarum ac mirabilium rerum in eis contentarum diligentissime elaborata. Anno Dni 1539, Venise, 1539. La carte, formée d'un assemblage de 9 feuilles, comporte un privilège pour dix ans accordé par le pape Paul III et signé Blosius, en date du 2 mars 1539. L'adresse est ainsi libellée :

"Venundantur in Apotheca Thome de Rubis in corona super ripam ferri prope Pontem rivi alti Venetiis. » En bas à droite, à côté de la signature : « Olaus Magnus Gotus Lincopen[sis] », et sous la devise : «Vince in bono malum », une figure représente la fable du lion et du rat, accompagnée de cette légende : « En leo terribilis quem solvit mus laqueatum./ Sic Magni minima sepe iuvantur ope. »-J'ai utilisé le fac-similé de l'exemplaire de l'Universitetsbibliotek d'Uppsala, aussi bien que des clichés de l'exemplaire de Munich, Bayerische Staatsbibliothek : 12 Mapp. VII, 1.

19. Iliade, XVIII, 587-610.

20. Voir ici la contribution de Mireille Séguy.

\section{RÉSUMÉS}

Du Moyen Âge à la Renaissance, les îles présentent quatre propriétés principales : le dynamisme, l'aptitude à l'encyclopédie, l'ouverture verticale et la miniaturisation. En premier lieu, le monde clos du Moyen Âge s'est ouvert par ses îles périphériques, des Canaries aux Moluques et aux Antilles. Réserves de savoir et magasins de singularités, les îles sont aussi des sas ouvrant sur d'autres mondes, sur l'au-delà et les espaces mouvants de la légende ou du mythe. Les îles, enfin, introduisent des trous dans le récit, favorisant la mise en abyme, la réflexivité et l'ironie. L'Utopie de Thomas More est la meilleure illustration de cette voie ironique des îles.

The Pathway of Islands. From the Middle Ages to the Renaissance, islands offered four main characteristics : dynamism, availability to encyclopaedic knowledge, vertical openess and a world in miniature. In the first place, the closed world of the Middle Ages opened itself to the islands located on its peripheries, the Canaries, the Moluccas and the West Indies. Warehouses of knowledge and stores of singularities, islands are also gateways to other worlds, the hereafter and the moving sands of myth and legend. Finally, islands introduce gaps in the narrative, thus favourising reflexivity and irony. Thomas More's Utopia is the best illustration of this ironic pathway of islands. 
INDEX

Mots-clés : archipel, atlas, Monde ouvert, Moyen Âge et Renaissance, Purgatoire, Utopie, Isolari Keywords : archipelago, atlases, ekphrasis, Open world, Middle Age and Renaissance, Purgatory, Utopia

\section{AUTEUR}

\section{FRANK LESTRINGANT}

Université Paris IV-Sorbonne, UFR de Lettres, 1, rue Victor Cousin, F-75230 Paris Cedex 05 